\title{
Retinal microscope
}

\author{
G. MAXWELL STUBBS \\ From Melbourne, Victoria, Australia
}

Increasing awareness of retinal degeneration and its significance in the development of retinal detachment has resulted in much more use of indirect ophthalmoscopy, which allows a larger field of view of the fundus and some variation in magnification depending on the power of the condensing lens used. A low power condensing lens provides a larger image, but with conventional instruments this is very unstable. It is usually necessary to accept a smaller image which does not suddenly disappear with slight movements of the lens. A retinal microscope has been developed which overcomes these disadvantages.

\section{Specification}

The instrument (Figs $1,2,3$ ) provides a stable image of apparent magnification equivalent to that of the direct ophthalmoscope, but with a field of 6 to 7 disc diameters and with all the advantages of

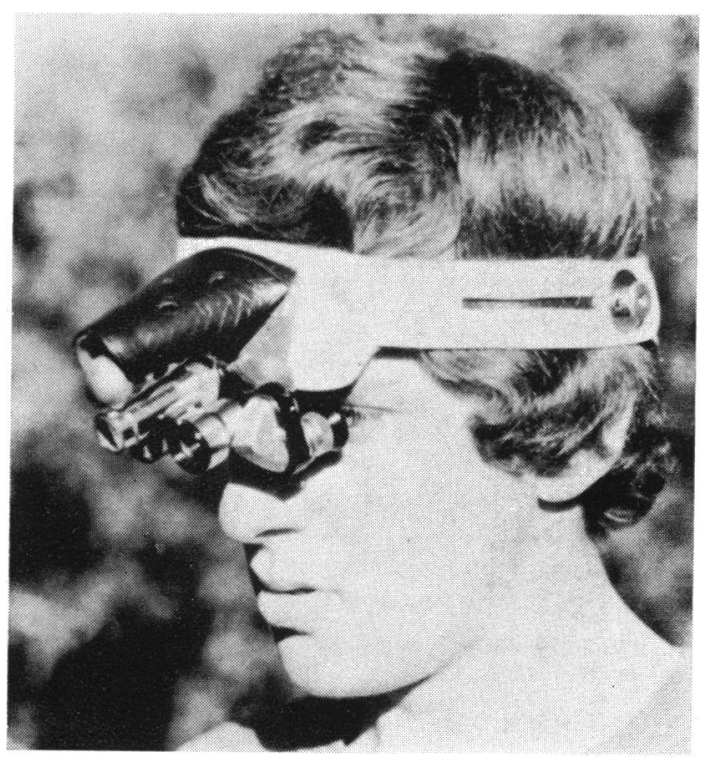

FIG. I Microscope in position

Address for reprints: Dr G. Maxwell Stubbs, 3rd Floor, 2 Collins Street Melbourne 3000, Australia

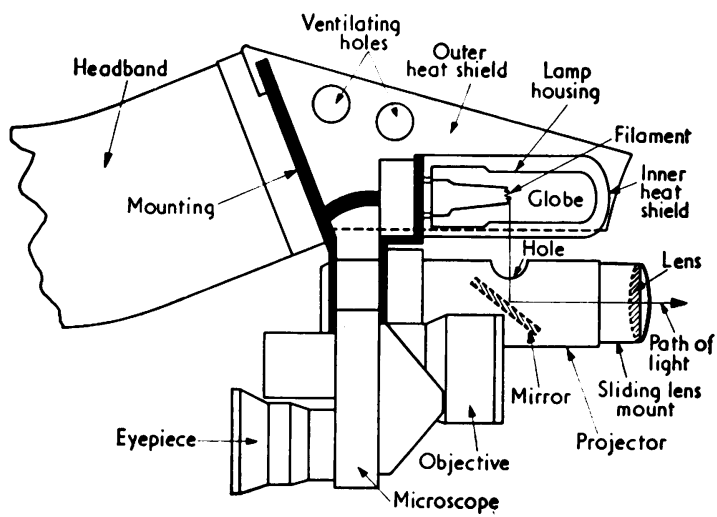

FIG. 2 Diagram of longitudinal section of instrument

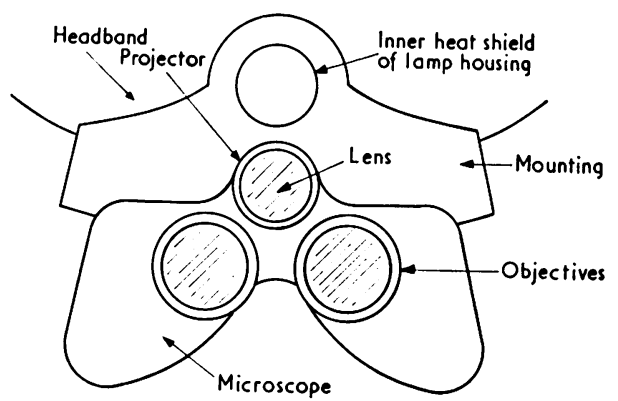

FIG. 3 Diagram of frontal view

binocular viewing. Resolution of the image and stability are more than adequate for routine use in the consulting room or operating theatre. Furthermore, on discarding the condensing lens an operating microscope of $6 x$, comfortably worn on the head, is immediately available. Thus the need for frequent change from indirect ophthalmoscope to operating loupe is obviated. No adjustment is needed, and from my experience of two years' use the magnification is not too great for the ordinary buckling, scleral resection, and other detachment procedures. An adequate depth of focus is obtained.

This arrangement allows detailed examination of areas of retinal degeneration, a difficult task with 


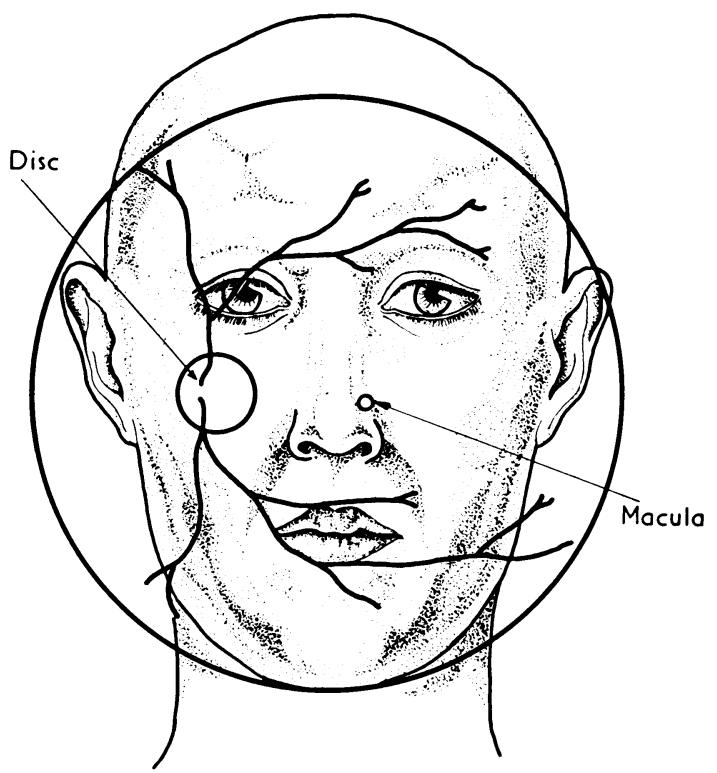

FIG. 4 Approximate size of fundal image using $20 D$ condensing lens

conventional indirect instruments, and the great advantage of mobility when used as an operating microscope. The lamp filament may be focused for the increased illumination needed for anterior chamber surgery, but a more diffuse patch of light is quickly obtainable by adjusting the focus, and this provides even illumination of the fundus during ophthalmoscopy. This is possible because of the intense light source of $5 \circ \mathrm{W}$, and it eliminates the annoying patchy illumination produced by conventional instruments. A $20 \mathrm{D}$ condensing lens gives bests results (Fig. 4). The quartz iodine lamp produces considerable heat, but suitable ventilation and insulation has been achieved.

The instrument is particularly useful in the consulting room, especially when the condensing lens is held close to the eye. Detailed examination of the cornea and anterior chamber is simply done, and even gonioscopy is possible, with a simultaneous view of the entire angle.

\section{Summary}

An instrument is described that provides a stable magnified image for ophthalmoscopy and a low power operating microscope combined to allow greatly improved fundal inspection and to eliminate the need to change instruments during detachment surgery.

I thank $\mathrm{Mr}$ Lorimer Fis $\mathrm{n}$ and OPSM Melbourne for their help and advice.

The production is currently in the hands of Keeler Instruments Ltd. 